\title{
¿CÓMO AFECTAN LOS MEDIOS SOCIALES A LA CONFIDENCIALIDAD DE LOS PACIENTES? REVISIÓN DE LOS POTENCIALES PROBLEMAS Y RECOMENDACIONES
}

\begin{abstract}
Cristina Ma Beltrán Aroca ${ }^{1}$, Eloy Girela López ${ }^{1}$
Resumen: Los datos personales relativos a la salud de un individuo se consideran como datos sensibles, por lo que necesitan una "especial protección". Nos encontramos en una sociedad en la que las tecnologías de información y comunicación (TIC), especialmente en lo referente a las redes sociales, ofrecen amplias posibilidades de intercambio de información médica, sirviendo de apoyo, consejo o ayuda a los compañeros que se encuentran en el mismo ámbito o en cualquier lugar de la geografía mundial. Los avances tecnológicos en comunicación desafían el concepto de "público" y de "privado", de tal forma que la información que se comparte es casi imposible de eliminar, pudiendo llegar a propagarse rápidamente por las redes. En base a nuestra propia experiencia y a los diferentes trabajos revisados, creemos que resulta necesario capacitar a los profesionales de la salud y especialmente a los estudiantes de medicina durante su formación, para que utilicen de forma adecuada dichas herramientas, especialmente a la hora de publicar contenido sensible en lugares cuyo acceso sea público, ya que puede tener consecuencias importantes para la intimidad de las personas, tanto para los pacientes como para su propia vida personal.
\end{abstract}

Palabras clave: confidencialidad, medios sociales, redes sociales, estudiantes, teléfonos móviles, secreto médico, intimidad, datos sanitarios

\section{How social media affects the confidentiality of patients? A review of potential problems and recommendations}

\begin{abstract}
Personal health data are considered as sensitive information, so they need a "special protection". We are in a society where Information and Communications Technology (ICT), especially in relation to social networks, offer many opportunities for the exchange of medical information, serving as support, advice or assistance to colleagues who work in the same field or in any other place around the world. Technological advances in communication challenge the concept of "public" and "private," in a way that the shared information is almost impossible to remove, and could spread rapidly through social networks. Based on our own experience and the different papers reviewed, we believe it is necessary to train health professionals and especially medical students during their academic education to use these tools properly, especially when posting sensitive data in places of public access, as it may have important consequences for the privacy of individuals, both for patients and for their own life.
\end{abstract}

Key words: confidentiality, social media, social networks, students, smartphones, medical secrecy, privacy, health data

\section{Como mídias sociais afetam a confidencialidade dos pacientes? Revisáo dos potenciais problemas e recomendaçóes}

Resumo: Os dados pessoais relativos à saúde do indivíduo são considerados dados sensíveis, por esse motivo eles necessitam de uma "proteção especial". Nos encontramos em uma sociedade em que as tecnologias de informação e comunicação (TIC), especialmente em relação às redes sociais, oferecem amplas oportunidades para a troca de informaçôes médicas, servindo como apoio, conselho ou assistência aos colegas que estáo na mesma área ou em qualquer lugar na geografia mundial. Os avanços tecnológicos em comunicação desafiam os conceitos de "público" e de "privado", de tal maneira que a informação compartilhada é quase impossível de ser eliminada, podendo se propagar rapidamente através das redes. Com base em nossa própria experiência e em diferentes trabalhos revisados, acreditamos que é necessário capacitar profissionais de saúde e especialmente os estudantes de medicina durante sua formação, para que utilizem de forma adequada estas ferramentas, especialmente quando se trata de publicar conteúdo sensível em locais cujo acesso será público, uma vez que pode ter implicaçôes importantes para a privacidade das pessoas, tanto para pacientes como para sua própria vida pessoal.

Palavras-chave: confidencialidade, meios de comunicação sociais, redes sociais, estudantes, telefones celulares, segredo médico, privacidade, dados de saúde

\footnotetext{
${ }^{1}$ Unidad Docente de Medicina Legal y Forense. Facultad de Medicina y Enfermería. Universidad de Córdoba, España Correspondencia: cristinabeltran@uco.es
} 


\section{Datos sensibles. Protección de datos relaciona- dos con la salud}

Cuando se hace referencia a la protección de datos, estos no se limitan a los íntimos, sino a cualquier tipo de dato personal, cuyo conocimiento o empleo por terceros pueda afectar derechos, porque su objeto es la protección de todos los datos de carácter personal. Por tanto, no tan solo son los datos de la vida privada o íntima de la persona, sino que se amparan todos los que identifiquen o permitan la identificación de la persona, pudiendo servir para la confección de su perfil privado o para otra utilidad que en determinadas circunstancias pudiera constituir una amenaza para el individuo(1).

Se entiende como datos personales, aquellos que se refieren a toda la información sobre una persona natural identificada o identificable (persona concernida). Una persona física no será considerada como "identificable" si dicha identificación necesitare plazos y actividades poco razonables. Si una persona natural no fuere identificable, los datos se considerarán anónimos. Cuando los datos de carácter personal revelen la raza de origen, ideas políticas, creencias religiosas u otras convicciones, al igual que los relativos a la salud, a la vida sexual, a condenas penales, así como otra información definida como sensible según la legislación nacional, se considerarán datos sensibles(2).

Los datos sensibles necesitan una "especial protección”, por lo que hay que extremar las precauciones, ya que afectan a lo más íntimo y reservado de los seres humanos. Es evidente que nunca podrá haber una protección absoluta, pero hacemos hincapié en que a los datos sanitarios hay que aplicar toda la protección que la sociedad y los individuos sean capaces. Se recogen como datos médicos todos aquellos datos personales relativos a la salud de un individuo, que tengan una clara y estrecha relación con la salud, y los datos genéticos(3). El perjuicio asociado con la revelación de estos datos puede ser financiero, de tal manera que su divulgación puede conducir a la negación de la cobertura de un seguro, pérdida del empleo o incluso falta de ofertas de trabajo. También puede afectar desde el punto de vista psicosocial, llegando a estigmatizar a una persona en su círculo social o en el trabajo, lo que puede provocar el aislamiento del sujeto o cierta dificultad para conseguir un empleo(4).

La calificación de datos sensibles necesitados de especial protección es no solo la consecuencia de la mayor sensibilidad de la sociedad actual para todas las cuestiones relacionadas con la intimidad personal, sino también, como veremos más adelante, del gran avance tecnológico, que ha hecho posible la vulneración de ese derecho por medios muy difíciles de detectar y que por ello mismo la mayoría de las veces quedan impunes(5). Se trata de un peligro potencial, derivado de la difusión de datos a escala mundial a través de internet, así como la posibilidad de su conocimiento por un número ingente de personas, a lo que se añade la dificultad de eliminar esos datos de la web ("derecho al olvido").

Tal como recoge Navarro, entre las soluciones para la publicación y protección de datos se podría proponer cualquier tipo de proceso que dificulte la identificación de determinadas características. En términos generales, se plantean técnicas que codifiquen o encripten los datos, también la omisión o generalización, la realización de una seudo anonimización de los mismos, la mezcla o reorganización de los diferentes campos, así como la aplicación de controladores de consultas en las bases de datos(6).

Los profesionales sanitarios están obligados a proteger estos datos especialmente sensibles de los pacientes, y para ello la legislación vigente de cada país y los códigos deontológicos que rigen la profesión médica los salvaguardan mediante el secreto profesional.

\section{Evolución del secreto profesional en el ejerci- cio de la medicina}

Altisent define la confidencialidad como "el derecho moral que asiste a las personas para que se mantenga la reserva sobre lo que confían a otros, quienes de manera correlativa adquieren la obligación de guardar secreto" (7). Como señala Diego Gracia, el secreto profesional en el ámbito de la medicina ha ido evolucionando con el paso de los años, de tal forma que en épocas antiguas la idea predominante era que el médico, como otros profesionales, debía guardar el secreto como de- 
ber de discreción y sigilo(8). Ello queda claro en el Juramento Hipocrático, máximo exponente del pensamiento médico de esta época: "Cualquier cosa que vea u oiga en el curso del tratamiento, o incluso fuera del tratamiento, que haga referencia a la vida de los hombres, que en ningún caso deba ser divulgada, la guardaré para mí mismo sin hablar nunca de ella". En la actualidad, en medicina, el secreto es relativo y se entiende como la obligatoriedad de guardar el secreto por parte del médico respecto del estado de salud y confidencias del paciente, pero siempre que no se perjudique con ello a los demás o a los intereses sociales o generales(9).

Desde esta perspectiva, el secreto médico es más vago y plantea más problemas en la práctica, resultando a veces difícil su aplicación(10), especialmente teniendo en cuenta que hoy asistimos a un cambio social muy intenso. Nos encontramos en una sociedad en la que la privacidad tiende a desaparecer, el derecho a la imagen y la propia intimidad son conceptos que se diluyen a favor de una cultura de hiperconexión y apertura de imagen y contenidos. Por otra parte, las técnicas informáticas, especialmente las redes sociales, ofrecen amplias posibilidades de intercambio de información médica, sirviendo de apoyo, consejo o ayuda a los miles de compañeros que se encuentran en el mismo ámbito o en cualquier lugar de la geografía mundial(11).

\section{Nuevas tecnologías y confidencialidad de los pacientes: ¿dónde se encuentran los límites?}

El uso de los medios sociales se ha generalizado y ofrece a los profesionales sanitarios y las organizaciones de la salud nuevas oportunidades de interactuar con los pacientes, el público, y los responsables políticos entre otros(12). En el ámbito sanitario, los profesionales han comenzado a utilizar las tecnologías digitales para beneficiar a los pacientes y mejorar la comunicación con ellos, mejorar el desarrollo y las redes profesionales y avanzar en la comprensión de los factores individuales y contextuales que influyen en la salud pública. Sin embargo, el debate de los peligros de estas tecnologías en la medicina ha sobrepasado a las aplicaciones positivas(13).
En el momento actual, entre las alternativas a la forma tradicional de ejercer la medicina en consulta, hay evidencias que apuntan al uso extendido de la consulta por teléfono por parte de los pacientes para obtener resultados de pruebas y otro tipo de información médica, sobre todo en el ámbito de la atención primaria(14). Así, se permite a los pacientes y familias estar informados de manera rápida y sin salir de casa. El principal inconveniente se centra en que la mayoría de estas herramientas (historia clínica informatizada, consultas por internet, recetas electrónicas, recetas online, telemedicina) están enfocadas principalmente en los aspectos tecnológicos, clínicos y económicos; sin embargo, no se han realizado avances conjuntos en relación con los aspectos éticos y legales, para proteger la confidencialidad de los pacientes(15). Respecto de las consultas telefónicas, si se proporciona información a un paciente o familiar sin identificación, se deja abierta la posibilidad de que personas no autorizadas, como amigos, curiosos, familiares o periodistas, puedan llamar y obtener información confidencial con bastante facilidad(16). Mientras que, por otra parte, si el médico se niega categóricamente a revelar información a través del teléfono, aunque protege la confidencialidad del paciente, se lo puede estar prohibiendo a alguien que tiene acceso legítimo a la misma. Por ello, como alternativa, se propuso la posibilidad de ofrecer a quien lo solicitara, un sistema de acceso que requiera contraseñas preestablecidas y conocidas solo por las personas autorizadas por el paciente(17).

Por otro lado, encontramos los medios sociales, entre los que cabe destacar las redes sociales como Facebook, YouTube, Twitter, MySpace, etc., que permiten a millones de personas en todo el mundo establecer contactos y compartir la información personal y profesional, fotografías, videos y blogs(18). En el caso concreto de Facebook, que cuenta con 1.44 billones de usuarios, el acceso a información, como el nombre, foto de perfil o vínculos personales, está disponible para todo el mundo a través de Internet (incluyendo personas no registradas en Facebook) utilizando los diferentes motores de búsqueda, pudiendo así importar o exportar dichos datos sin límites de privacidad(19). Es algo que puede resultar increíblemente útil pero, a la vez, constituye un riesgo 
potencial, ya que facilita que cualquier otra persona pueda también compartir nuestra información, o bien que los datos o fotos que hayamos publicado queden para siempre online, y en el caso de que sea información personal sobre la salud, podría llegar a constituir un serio problema(20). A excepción de las restricciones claras, que se relacionan con la protección de derechos de autor y la actividad criminal, en la mayoría de las redes sociales la configuración de privacidad es variable y es controlada por el usuario de manera personalizada(21).

En relación con los datos sobre la salud, la accesibilidad y la "indexabilidad" de la información publicada en línea origina nuevos problemas para el mantenimiento de la confidencialidad, tratándose de un aspecto directamente relacionado con el consentimiento informado del paciente respecto a la disposición de sus datos(22). Estudios posteriores corroboran que, efectivamente, uno de los problemas éticos principales que plantean las nuevas tecnologías continúa siendo el mantenimiento de la privacidad y confidencialidad de los usuarios, lo cual se corresponde con tres de los principios fundamentales de la ética médica: autonomía, beneficencia y justicia. Al aplicar estos principios en el contexto de los medios sociales y la divulgación de datos sanitarios y salud pública, es necesario insistir en que cada persona tiene derecho a decidir sobre el uso de aquellos datos relativos a su vida privada - derecho de autodeterminación-. Por otra parte, en cuanto a los principios de beneficencia y justicia, se requiere la eficacia y disponibilidad de medios que permitan la detección de aquellas situaciones en las que se ponga en peligro la confidencialidad(23).

Debemos tener en cuenta que, en el caso de las redes sociales, estas albergan personas que también son pacientes, lo que puede conllevar conflictos en una doble vertiente. Por una parte, en relación a la vida privada del propio médico, en ocasiones puede resultar difícil establecer límites entre el ámbito personal y profesional del mismo. Mientras que la otra vertiente del conflicto hace referencia a que, en aquellas páginas o grupos de redes sociales como Facebook, en los que se consulta o facilita información médica, se podría plantear como uno de los principales problemas éticos la confidencialidad de los datos de los pacientes, bien porque estos no se almacenen de forma segura y confidencial por parte del médico, o porque sean compartidos de forma poco apropiada entre los propios facultativos(24). Se trata en parte de un problema relacionado con la conducta de los profesionales sanitarios, y ante el cual no existen normas o límites legales establecidos por los responsables políticos en materia de salud, lo que genera cierto miedo individual e institucional ante el uso de los medios sociales en medicina(25). En un estudio realizado por Panahi et al., en el que se tuvo en cuenta la opinión de los facultativos, se puso de manifiesto que, aunque reconocían que la comunicación y el aprendizaje cara a cara en tiempo real eran insustituibles, resultaba positivo participar en las redes sociales para mantenerse al día en cuanto a formación, así como intercambiar conocimientos o establecer contacto con otros compañeros en la distancia. Por otra parte, los principales retos que les planteaba el uso de tales tecnologías estaban relacionados con la confidencialidad de los pacientes, entre otros, como la falta de participación activa o incluso de tiempo para dicha actividad(26). Siguiendo esta línea, se describió un caso en que el paciente no podía facilitar información sobre su patología debido a su situación clínica, y el equipo médico llegó a utilizar datos personales procedentes de un perfil de Facebook para prestarle atención médica(27).

Posteriormente se recogieron otras utilidades, como por ejemplo personas allegadas a un enfermo, que también se valieron de este medio para difundir e identificar posibles contactos de enfermedades infecto-contagiosas(28). En el primer caso se justificó la actuación a favor del principio de beneficencia, y en el segundo la protección de la salud pública sobre la autonomía y confidencialidad. En 2015, un estudio en relación a la búsqueda en línea de información sobre pacientes por parte de médicos y estudiantes(29) puso de manifiesto que hasta un 13,5\% de los participantes había buscado en Google datos sobre los enfermos, y un 2,1\% en Facebook, aunque dicha práctica la realizaban por igual ambos colectivos. La búsqueda de información sobre un paciente en buscadores o redes sociales podría ser una versión más moderna, semejante a buscar datos en su propia carpeta cuando presenta un nivel alterado de conciencia. Por otra parte, si bien es cierto que 
una búsqueda en línea solo revelará información que ya es de acceso público, puede causar problemas respecto del consentimiento y la exactitud de la información del paciente.

Hay que destacar el caso de los médicos más jóvenes - residentes-, entre los cuales está ampliamente extendido el uso de las redes sociales ( $\mathrm{Fa}$ cebook, Linkedin y Twitter), sitios web para compartir información (Flickr y YouTube), así como blogs, wikis, podcasts y teléfonos móviles, por lo que tiene especial relevancia. Existen evidencias que apuntan a que en este colectivo también se dan comportamientos poco profesionales, así como conflictos en relación a la confidencialidad, tanto en la información personal de sus propias cuentas, como respecto de los pacientes(30).

En una revisión sistemática de diferentes publicaciones, se puso de manifiesto que los estudiantes de medicina (64-96\%) tenían perfil en Facebook y utilizaban herramientas como Wikipedia, incluso con más frecuencia que los residentes y el resto de médicos (13-47\%), aunque prácticamente de la misma forma, tanto los estudiantes $(62,1 \%)$ como los residentes $(67,5 \%)$ tenían perfiles públicos en sus cuentas de las redes sociales (32). En 2009, Chretien et al. encuestaron a decanos de diferentes facultades de medicina en EEUU y mostraron que en el 60\% de las mismas (47/78) ocurrían incidencias en relación a conductas poco profesionales por parte de los estudiantes, y hasta en un $13 \%$ se daban situaciones en las que se vulneraba la confidencialidad de los pacientes(33). Posteriormente, Thompson et al. realizaron un estudio en el que, analizando los perfiles de Facebook en un grupo de médicos residentes y estudiantes, comprobaron que se violaba la confidencialidad de los pacientes, mediante la publicación de fotografías durante viajes a países en vías de desarrollo en misión humanitaria, hecho que ocurría especialmente entre los estudiantes (11/12 situaciones)(34).

Otro aspecto importante relacionado con las nuevas tecnologías y que también ha sido descrito especialmente en los estudiantes de medicina, es el uso de los teléfonos móviles. Los smartphones se están convirtiendo en un elemento omnipresente en el ámbito de la atención sanitaria. El aumento del uso de la tecnología móvil — teléfonos inte- ligentes - se puede atribuir a las mejoras percibidas en la comunicación clínica, la eficiencia y las habilidades clínicas(35). Estos smartphones también están siendo reconocidos por su utilidad en la educación médica y en la formación, de ahí que se presenten como un recurso que permite "aprender en cualquier lugar" (36). Tanto los estudiantes de medicina como los médicos están utilizando aplicaciones médicas con finalidad educativa y clínica, y las más populares son las relacionadas con fármacos de referencia, diagnóstico y manejo de diferentes patologías, así como escalas de puntuación clínica(37). Además de las ventajas que proporcionan los smartphones, el hecho de que dispongan de cámaras fotográficas resulta especialmente peligroso, al poder tomar imágenes de lesiones de pacientes con cualquier finalidad. Pueden ser utilizadas para consultar aspectos meramente clínicos con otros compañeros, o recopiladas como material docente; pero también se pueden compartir a través de aplicaciones de mensajería instantánea, como Whatsapp, o bien quedar almacenadas en el teléfono, sin poder controlar el acceso al mismo por parte de otros usuarios no sanitarios y no autorizados para observarlas. Este hecho lo pudimos comprobar mediante un trabajo de investigación que planteamos en un hospital de referencia de tercer nivel, en relación a la observación de faltas a la confidencialidad cometidas por el personal sanitario durante su labor asistencial. Mediante observaciones recogidas por los estudiantes de medicina, previo entrenamiento, se puso de manifiesto que, en ocasiones, todo tipo de profesionales sanitarios utilizaban los teléfonos móviles para enviar imágenes o datos clínicos de casos destacados de pacientes a otros compañeros o incluso familiares y amigos. Un hallazgo significativo fue el grupo de los estudiantes, en los que se observó esta conducta relacionada con los teléfonos móviles en un $14,3 \%$ de las faltas a la confidencialidad que cometieron en total (1/7) (resultados no publicados). Un estudio realizado por Tran et al., en 2014, mediante entrevistas a los estudiantes acerca del uso del teléfono móvil en el entorno clínico, mostró que la gran mayoría de ellos poseían un smartphone (98\%, 97/99), y que además lo utilizaba para temas relacionados con los pacientes durante las rotaciones clínicas (86\%, 85/99)(38). Reconocían que, gracias al uso de estos dispositivos, el trabajo clínico era 
más eficiente $(94 \%, 90 / 96)$. Por el contrario, hasta un 68\% (65/95) estaba de acuerdo en que utilizar los teléfonos personales para compartir con los compañeros información relacionada con los pacientes, y un 22\% (21/96) respecto a los datos de identificación de los mismos, suponía un riesgo para la privacidad y confidencialidad. Recientemente, otro trabajo puso de manifiesto la preocupación por la falta de seguridad en la transmisión de datos sensibles de los pacientes a través de Whatsapp. Si bien también se evidenciaron beneficios en la comunicación clínica entre el personal del equipo sanitario e incluso con los propios estudiantes durante su formación en el hospital(39).

El problema de la vulneración de la confidencialidad de los pacientes en relación a los avances tecnológicos afecta tanto a los profesionales sanitarios como a los estudiantes de medicina, en los que resulta de especial importancia y preocupación. Por ello, en 2010, Kind et al. analizaron si estos recibían algún tipo de instrucción o normativa por parte de las propias facultades de medicina para un uso correcto de dichos medios. Mediante su estudio informaron que solo 13 de 132 facultades en Estados Unidos tenían directrices explícitas acerca del manejo de las nuevas herramientas. En el caso de aquellas que sí tenían normas específicas, hacían referencia a comportamientos que deberían estar prohibidos, a aquellos que estaban desaconsejados y también indicaban los que eran adecuados a la hora de utilizar los medios sociales, todo ello con el fin de ayudar a los estudiantes(40).

\section{Recomendaciones}

Las evidencias recogidas nos muestran los problemas éticos que derivan del uso inadecuado de las tecnologías digitales, en concreto aquellos relacionados con la confidencialidad de los pacientes. Sobre la base de nuestra propia experiencia y las propuestas de diversos autores, a continuación detallamos una serie de recomendaciones o posibles soluciones que deben tener en cuenta los profesionales sanitarios para utilizar dichas herramientas de manera ética y segura $(6,16,28,33,41-43)$ :

Aunque se considera que las consultas por teléfono pueden ser una herramienta útil en la práctica clínica, como medida de seguridad se recomienda implementar un sistema de contraseńas que evite casos de suplantación y, por tanto, la puesta en peligro de la confidencialidad.

Para mantener la privacidad del médico en las redes sociales es conveniente separar las cuentas o páginas dedicadas a tratar con los pacientes y su actividad clínica, de aquellas que tengan contenido relacionado con su vida personal.

Respetar y mantener los límites de la relación médico-paciente, de acuerdo con las normas éticas profesionales. Esto puede resultar un reto cuando un médico recibe una solicitud de amistad de un paciente, por lo que se recomienda evitar aceptarlas.

Establecer la configuración de privacidad adecuada para asegurar que el contenido cuyo acceso es privado no se haga público.

Se recomienda decidir con cautela el contenido que se va a publicar, teniendo en cuenta que puede convertirse en público o ser compartido por los pacientes y compañeros. Además, se mantiene en la red por mucho tiempo. En muchas ocasiones, determinadas descripciones realizadas sobre un caso concreto o detalles sobre la historia del paciente pueden ser suficientes para identificarlo, considerando además que el uso de seudónimos no siempre es suficiente para mantener la confidencialidad.

En cuanto a las publicaciones de pacientes en redes sociales, es importante obtener el consentimiento de los mismos, e incluso se deben intentar buscar otras formas diferentes a la escrita en aquellos casos que sea necesario.

Respecto de las fotografías, es aconsejable utilizar programas de edición digital que modifiquen o distorsionen la imagen para evitar el reconocimiento del sujeto.

En determinadas ocasiones puede resultar necesaria la creación de grupos "privados" en las redes sociales, de tal forma que solo los pacientes pertenecientes al mismo puedan consultar la información.

En determinados casos se han utilizado las redes 
sociales como medio de comunicación para control de enfermedades transmisibles, ante lo que se recomienda valorar previamente las características de la enfermedad y el riesgo público sanitario real, frente a la confidencialidad de los pacientes en los medios sociales.

En cuanto los estudiantes, se recomienda formación específica por parte de las facultades de medicina, para que utilicen adecuadamente, desde el punto de vista práctico y ético, las herramientas digitales y teléfonos móviles personales en la comunicación clínica.

\section{Conclusiones}

El futuro de las nuevas herramientas digitales en el ámbito de la salud es prometedor. Las redes sociales pueden llegar a revolucionar la medicina en relación a consultas clínicas, en cuanto a la interacción entre profesionales, al intercambio de conocimientos y la democratización de la salud, al permitir que más personas (incluyendo pacientes, otros profesionales clínicos, medios de comunicación) puedan opinar y participar en las diversas actividades médicas relacionadas.

Los avances tecnológicos en comunicación desafían el concepto de "público" y de "privado", de tal forma que la información que se comparte es casi imposible de eliminar, pudiendo llegar a propagarse rápidamente por las redes. Esto no quiere decir que los profesionales médicos deban evitar el uso de los medios sociales, ya que tanto su utilidad personal como profesional puede ser beneficiosa. Para ello es necesario capacitar a los profesionales de la salud y a los estudiantes de medicina en formación, para que utilicen de forma adecuada dichas herramientas, especialmente a la hora de publicar contenido sensible en lugares cuyo acceso sea público, ya que puede tener consecuencias importantes para la intimidad de las personas, tanto para los pacientes como para su propia vida personal. 
¿Cómo afectan los medios sociales a la confidencialidad de los pacientes? - Cristina Beltrán Aroca, Eloy Girela López

Referencias

1.

Sentencia 292/2000, de 30 de noviembre de 2000. Recurso de inconstitucionalidad 1.463/2000. Promovido por el Defensor del Pueblo respecto de los arts. 21.1 y 24.1 y 2 de la Ley Orgánica 15/1999, de 13 de diciembre, de Protección de Datos de Carácter Personal. Vulneración del derecho fundamental a la protección de datos personales. Nulidad parcial de varios preceptos de la Ley Orgánica. Pleno del Tribunal Constitucional. Boletín Oficial del Estado, no 4, (4 enero 2001).

2. Consejo de Europa. Recomendación No R(97) 18, y exposición de motivos del Comité de Ministros a los Estados miembros relativa a la Protección de datos de carácter personal, recogidos y tratados con fines estadísticos (adoptada por el Comité de Ministros el 30 de septiembre de 1997, en la 602 reunión de Delegados de Ministros).

3. Consejo de Europa. Recomendación $n^{\circ} R(97)$ 5, de 13 de febrero de 1997, del Comité de Ministros del Consejo de Europa a los Estados miembros sobre Protección de datos médicos.

4. Lane J, Schur C. Balancing Access to Health Data and Privacy: A Review of the Issues and Approaches for the Future. Health Serv Res 2010; 45 (5 Pt 2): 1456-1467.

5. Organización Médica Colegial de España. Guías de ética en la práctica médica. Intimidad, confidencialidad y secreto. Madrid: Fundación de Ciencias de la Salud; 2005.

6. Navarro R. An ethical framework for sharing patient data without consent. Inform Prim Care 2008; 16(4): 257-262.

7. Altisent R. Confidencialidad. En: Romeo CM, ed. Enciclopedia de Bioderecho y Bioética. Granada: Comares S.L.; 2011: 425-430.

8. Gracia D. Fundamentos de bioética. 2a ed. Madrid: Triacastela; 2007.

9. Castellano M, Gisbert JA. El secreto médico. Historia clínica. Confidencialidad y otros problemas médico-legales de la documentación clínica. En: Villanueva E, ed. Gisbert Calabuig. Medicina legal y toxicología. 6a ed. Barcelona: Masson, S.A.; 2004: 93-108.

10. Criado MT, Seoane J. Aspectos médico-legales de la historia clínica. Madrid: S.A. Colex. Editorial Constitución y Leyes; 1999.

11. Organización Médica Colegial de España. Ética y Redes Sociales. Manual de estilo para médicos y estudiantes. Madrid: OMC; 2014.

12. Patel D, Jermacane D. Social media in travel medicine: a review. Travel Med Infect Dis 2015; 13(2): 135-142.

13. George DR, Rovniak LS, Kraschnewski JL. Dangers and opportunities for social media in medicine. Clin Obstet Gynecol 2013; 56(3): 453-462.

14. Patel H, Patel M, Car J. Telephone consultations in general practice: areas for improvement. Journal of Telemedicine and Telecare 2005; 11: 265-266.

15. Casado M. Ética y legalidad en el uso de tecnologías de la información. Diario médico.com. 4 mayo 2015. [Consultado 18 junio 2015]. Disponible en http://www.diariomedico.com/2015/05/04/opinion-participacion/columnainvitada/etica-y-legalidad-uso-tecnologias-informacion.

16. Sokol D, Car J. Protecting patient confidentiality in telephone consultations in general practice. Br J Gen Pract 2006; 56(526): 384-385.

17. Sokol D, Car J. Patient confidentiality and telephone consultations: time for a password. J Med Ethics 2006; 32: 688689.

18. McCreary L. What was privacy? Harv Bus Rev. 2008; 86(10): 123-130.

19. Statista. The statistics portal. Number of monthly active Facebook users worldwide as of 1st quarter 2015 (in millions). 2015 [Consultado 16 junio 2015]. Disponible en http://www.statista.com/statistics/264810/number-of-monthlyactive-facebook-users-worldwide/

20. O'Connor D. The apomediated world: regulating research when social media has changed research. J Law Med Ethics 2013; 41(2): 470-483.

21. Thackeray R, Neiger BL, Smith AK, Van Wagenen SB. Adoption and use of social media among public health departments. BMC Public Health 2012; 12: 242.

22. Mansfield SJ, Morrison SG, Stephens HO, Bonning MA, Wang SH, Withers AH, et al. Social media and the medical profession. Med J Aust 2011; 194(12): 642-644.

23. Denecke K. Ethical aspects of using medical social media in healthcare applications. Stud Health Technol Inform 2014; 198: 55-62.

24. Ahmed OH, Sullivan SJ, Schneiders AG, Anderson L, Paton C, McCrory PR. Ethical considerations in using Facebook for health care support: a case study using concussion management. PM R. 2013; 5(4): 328-334.

25. Grajales FJ, Sheps S, Ho K, Novak-Lauscher H, Eysenbach G. Social media: review and tutorial of applications in medicine and health care. J Med Internet Res 2014; 16(2): e13. 
26. Panahi S, Watson J, Partridge H. Social media and physicians: Exploring the benefits and challenges. Health Informatics J 2014 Jul 18. pii: 1460458214540907.

27. Ben-Yakov M, Snider C. How Facebook saved our day! Acad Emerg Med 2011; 18(11): 1217-1219.

28. Mandeville KL, Harris M, Thomas HL, Chow Y, Seng C. Using Social Networking Sites for Communicable Disease Control: Innovative Contact Tracing or Breach of Confidentiality? Public Health Ethics 2014; 7(1): 47-50.

29. Ben-Yakov M, Kayssi A, Bernardo JD, Hicks CM, Devon K. Do emergency physicians and medical students find it unethical to 'look up' their patients on Facebook or Google? West J Emerg Med 2015; 16(2): 234-239.

30. Baer W, Schwartz AC. Teaching professionalism in the digital age on the psychiatric consultation-liaison service. Psychosomatics 2011; 52(4): 303-309.

31. Von Muhlen M, Ohno-Machado L. Reviewing social media use by clinicians. J Am Med Inform Assoc 2012; 19(5): 777-781.

32. Thompson LA, Dawson K, Ferdig R, et al. The intersection of online social networking with medical professionalism. J Gen Intern Med 2008; 23: 954-957.

33. Chretien KC, Greysen SR, Chretien JP, Kind T. Online posting of unprofessional content by medical students. JAMA 2009; 302(12): 1309-1315.

34. Thompson LA, Black E, Duff WP, Paradise Black N, Saliba H, Dawson K. Protected health information on social networking sites: ethical and legal considerations. J Med Internet Res 2011; 13(1): e8.

35. Farnan JM, Snyder Sulmasy L, Worster BK, Chaudhry HJ, Rhyne JA, Arora VM, et al. Online medical professionalism: patient and public relationships: policy statement from the American College of Physicians and the Federation of State Medical Boards. Ann Intern Med 2013; 158(8): 620-627.

36. Trelease RB. Diffusion of innovations: smartphones and wireless anatomy learning resources. Anat Sci Educ 2008; 1(6): 233-239.

37. Payne KB, Wharrad H, Watts K. Smartphone and medical related App use among medical students and junior doctors in the United Kingdom (UK): a regional survey. BMC Med Inform Decis Mak 2012; 12: 121.

38. Tran K, Morra D, Lo V, Quan SD, Abrams H, Wu RC. Medical students and personal smartphones in the clinical environment: the impact on confidentiality of personal health information and professionalism. J Med Internet Res 2014; 16(5): e132.

39. Johnston MJ, King D, Arora S, Behar N, Athanasiou T, Sevdalis N, et al. Smartphones let surgeons know WhatsApp: an analysis of communication in emergency surgical teams. Am J Surg. 2015; 209(1): 45-51.

40. Kind T, Genrich G, Sodhi A, Chretien KC. Social media policies at US medical schools. Med Educ Online $2010 ; 15$. doi: 10.3402/meo.v15i0.5324.

41. Guseh JS, Brendel RW, Brendel DH. Medical professionalism in the age of online social networking. J Med Ethics 2009; 35: 584-586.

42. Leiker M. When to 'friend' a patient: social media tips for health care professionals. WMJ 2011; 110(1): 42-43.

43. Macintosh T. Ethical considerations for clinical photography in the global South. Dev World Bioeth 2006; 6(2): 81-88.

Recibido: 9 de octubre de 2015

Aceptado: 17 de octubre de 2015 